\title{
Basins of attraction: population dynamics with two stable 4-cycles
}

\author{
Shandelle M. Henson, R. F. Costantino, Robert A. Desharnais, J. M. Cushing and Brian Dennis
}

Henson, S. M., Costantino, R. F., Desharnais, R. A., Cushing, J. M. and Dennis, B.
2002. Basins of attraction: population dynamics with two stable 4-cycles. - Oikos 98: $17-24$

\begin{abstract}
We use the concepts of composite maps, basins of attraction, basin switching, and saddle fly-by's to make the ecological hypothesis of the existence of multiple attractors more accessible to experimental scrutiny. Specifically, in a periodically forced insect population growth model we identify multiple attractors, namely, two locally stable 4-cycles. Using the model-predicted basins of attraction, we examine data time series from a Tribolium experiment for evidence of the multiple attractors. We conclude that the multiple attractor hypothesis together with demographic stochasticity accounts for the experimental observations.
\end{abstract}

S. M. Henson, Dept of Mathematics, Andrews Univ., Berrien Springs, MI 49104, USA (henson@andrews.edu)._R.F.Costantino,Dept of Biological Sci., Univ. of Rhode Island, Kingston, RI 02881, USA. - R. A. Desharnais, Dept of Biology and Microbiology, California State Univ., Los Angeles, CA 90032, USA. - J. M. Cushing, Dept of Mathematics, Univ. of Arizona, Tucson, AZ 85721, USA. - B. Dennis, Dept of Fish and Wildlife Resources and Div. of Statistics, Univ. of Idaho, Moscow, ID 83844, USA.

The prospect of multiple stable states is a prediction of various nonlinear population models (May 1977, Kot and Schaffer 1984, Vandermeer and Yodzis 1999, King and Schaffer 1999, Roberds and Selgrade 2000). For example, mathematical models with free oscillations typically forecast multiple attractor phenomena when subjected to periodic forcing. In particular, discrete population models which cycle in the absence of timedependent factors generically develop multiple stable oscillatory solutions when perturbed with periodic forcing. In general the multiple cycles are out of phase and may differ in average (Henson 2000, Selgrade and Roberds 2001).

Under the experimental conditions of Desharnais and Costantino (1980) populations of the flour beetle Tribolium display 2-cycles when cultured in a constant habitat (Dennis et al. 1995). (A 2-cycle is a periodic solution with period two time units. In these experiments the unit of time equals the larval period of two weeks.) Mathematical theory predicts that, generically, a population with an inherent (i.e. constant habitat) stable 2-cycle will develop two distinct locally stable 2-cycles with the advent of period-2 forcing (Henson 2000). The latter prediction was empirically tested and confirmed in laboratory cultures of Tribolium (Henson et al. 1999).

Other fascinating theoretical forecasts are associated with other forcing periods. For example, when subjected to forcing of period 4 , a population with an inherent stable 2-cycle typically will develop two coexisting locally stable 4-cycles; and an unstable equilibrium will give rise to an unstable 4-cycle. In this paper, we test this hypothesis by analyzing experimental data collected in habitats forced with period 4. Our analysis confirms the hypothesis and, as a result, provides a previously unavailable explanation for the observed patterns in these data.

The multiple attractor case studied here differs from that studied by Henson et al. (1999) in several important ways. The period-2 study of Henson et al. (1999) involved experimental manipulations of demographic parameters and was designed explicitly to place the cultures in a multiple attractor regime. In the period- 4 experiment studied here, there are no such manipula-

Accepted 3 December 2001

Copyright (C) OIKOS 2002

ISSN 0030-1299

OIKOS 98:1 (2002) 
tions; the multiple attractors occur naturally. Furthermore, unlike the period-2 study there is no resonance phenomenon. In both studies, we draw on the conceptual tools of composite maps and basins of attraction from dynamical systems theory to connect the model predictions with the data.

\section{The data}

The time series records that we examine were obtained from an experiment conducted by Jillson (1980). Twelve cultures of the flour beetle Tribolium castaneum (Herbst) were initiated with 75 small larvae and 30 adults. The cultures were placed in $237 \mathrm{ml}$ milk bottles with $20 \mathrm{~g}$ of standard media (95\% flour, $5 \%$ dried brewer's yeast by weight) and kept in an unlighted incubator at $33^{\circ} \mathrm{C}$. Every 2 weeks the larvae, pupae and adults were counted, and returned to fresh media. Dead adults were counted and removed.

Following the census at week 18, six cultures were placed in a constant $20 \mathrm{~g}$ habitat and six were assigned to a period-4 habitat sequence of $32 \mathrm{~g}, 32 \mathrm{~g}, 8 \mathrm{~g}, 8 \mathrm{~g}, 32 \mathrm{~g}$, $32 \mathrm{~g}, 8 \mathrm{~g}, 8 \mathrm{~g}$ and so on (see Fig. 1). Note that the average flour volume in the period-4 habitat is the volume in the $20 \mathrm{~g}$ constant habitat cultures. (We take the unit of volume to be the volume occupied by $1 \mathrm{~g}$ of flour.) The cultures were maintained for a total of 70 weeks.

Time series plots of larval numbers for two of the cultures maintained in the period- 4 habitat after week 18 appear in Fig, 1. There are some notable similarities and differences between the cultures. In culture A (replicate \#6 in Jillson's experiment) larval numbers oscillate in a "low-high-low-high" fashion (in time with the $32 \mathrm{~g}-32 \mathrm{~g}-8 \mathrm{~g}-8 \mathrm{~g}$ flour volume oscillation) during the initial part and end of the experiment. The highest peaks occur during the second $32 \mathrm{~g}$ habitat (at weeks 20 ,

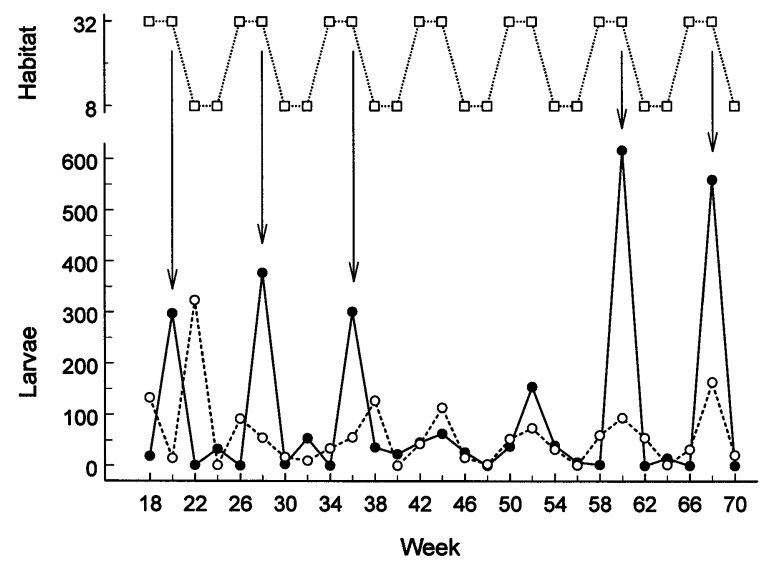

Fig. 1. Larval time series data of culture A (solid circles) and culture B (open circles) from the experiment of Jillson (1980) are shown. Habitat size (open squares) varies in the 4-cycle pattern: $32 \mathrm{~g}-32 \mathrm{~g}-8 \mathrm{~g}-8 \mathrm{~g}$.
28, 36 and at weeks 52,60,68). This temporal pattern is interrupted by an interlude between weeks 40 and 58 during which an entirely different pattern is observed.

Culture B (replicate \# 22 in Jillson's experiment), on the other hand, exhibits a "high-low-high-low" oscillation during the initial part of the experiment that is out-of-phase with culture A. Moreover, in contrast to culture A, culture B attains its highest peak larval numbers during the first $8 \mathrm{~g}$ habitat. However, like culture A, culture B also displays an interlude with a different oscillatory pattern, although for this culture the interlude is longer, lasting from week 28 to week 66 . By the end of the time series culture B appears to have attained an oscillation in-phase with culture A, attaining its peak larval number during the second week of the $32 \mathrm{~g}$ habitat.

In this paper we offer an explanation for the similarities and differences in the oscillatory patterns of these two cultures. Our explanation is based on the model predicted existence of three distinct 4-cycles, two of which are stable and one of which is unstable.

\section{The model}

The LPA (larva, pupa, adult) model is a system of three difference equations relating the numbers of animals at time $t+1$ to the number of animals at time $t$ :

$L_{t+1}=b A_{t} \exp \left(-c_{e l} L_{t}-c_{e a} A_{t}\right)$

$P_{t+1}=\left(1-\mu_{l}\right) L_{t}$

$A_{t+1}=P_{t} \exp \left(-c_{p a} A_{t}\right)+\left(1-\mu_{a}\right) A_{t}$

In this scheme (Dennis et al. 1995, 1997, 2001), $L_{t}$ is the number of feeding larvae (referred to as the L-stage), $P_{t}$ is the number of large larvae, pupae and callow adults (called P-stage), and $A_{t}$ is the number of sexually mature adults (A-stage animals) at time $t$. The unit of time is two weeks and is, approximately, the average amount of time spent in the feeding larval stage under our experimental conditions. The unit of time is also approximately the average duration of the P-stage. The quantity $b$ is the number of larval recruits per adult per unit of time in the absence of cannibalism. The fractions $\mu_{l}$ and $\mu_{a}$ are the larval and adult rates of mortality in one unit of time. The exponential nonlinearities account for the cannibalism of eggs by both larvae and adults, and the cannibalism of pupae by adults. The fractions $\exp \left(-c_{e l} L_{t}\right)$ and $\exp \left(-c_{e a} A_{t}\right)$ are the probabilities that an egg is not eaten in the presence of $L_{t}$ larvae and $A_{t}$ adults in one time unit. The fraction $\exp \left(-c_{p a} A_{t}\right)$ is the survival probability of a pupa in the presence of $A_{t}$ adults in one time unit. 
In order to explain deviations of data from predictions of the model equations (1a)-(1c) and to carry out statistical analyses for purposes of parameterization and validation, we construct a stochastic version of the LPA model containing demographic variability (Dennis et al. 2001). In the stochastic version, we used the binomial and Poisson distributions to describe the aggregation of demographic events within the life stages. Our stochastic demographic LPA model thus takes the conditional one-time-step distributions of $L_{t}, P_{t}$, and $A_{t}$ to be independent, discrete probability distributions.

For the parameter estimates and stochastic simulations reported in this paper, we transformed the observations so that the stochastic demographic LPA model would be well approximated by a nonlinear autoregressive (NLAR) model. A square root transformation of the state variables $L, P$ and $A$ accomplishes this approximation. The resulting NLAR model, in which noise is added to each state variable on the square root scale, can be regarded as a general method of incorporating demographic stochasticity into a deterministic model. The demographic nature of the stochasticity stipulates that the noise variables be uncorrelated through time as well as with each other within a time unit. We take the noise variables to be normally distributed with mean 0 . The resulting model has nine parameters, the deterministic LPA model parameters $b$, $\mu_{l}, c_{e a}, c_{e l}, c_{p a}, \mu_{a}$ and the three variances $\sigma_{1}, \sigma_{2}, \sigma_{3}$, of the noise variables. We used the method of conditional least squares to estimate these parameters from the four control (constant 20g habitat) cultures of Jillson (1980). The results are

$b=4.766, \mu_{l}=0.4087, c_{e a}=0.008462$,

$c_{e l}=0.04741, c_{p a}=0.01019, \mu_{a}=0.1513$,

$\sigma_{1}=7.267, \sigma_{2}=7.755, \sigma_{3}=0.4736$

For more details about the stochastic demographic LPA model see Dennis et al. (2001).

Mathematically the model defined by Eqns. (1a)-(1c) is called "autonomous". This means that time does not appear explicitly in the formulas, i.e., independently of the state variables $L_{t}, P_{t}$, and $A_{t}$. Ecologically this is a reflection of the fact that the model represents dynamics in a constant habitat where the demographic parameters $b, \mu_{a}, \mu_{l}, c_{e a}, c_{e l}$, and $c_{p a}$ are constant over time. This LPA model has been highly successful in explaining and predicting a variety of nonlinear patterns observed in data (Cushing et al. 1998b, Caswell 2000, Mueller and Joshi 2000). Therefore, it serves as an excellent foundation on which to base the construction of a model for populations in a fluctuating habitat. As we will see below, in a fluctuating habitat some of these parameters will fluctuate in time, in which case the model is said to be "nonautonomous". In the more specific case of a periodically fluctuating habitat some of the parameters will oscillate periodically in time, in which case the model is said to be "periodically forced".

In previous studies a modification of the autonomous LPA model proved extraordinarily successful in studies of flour beetle responses to habitat oscillations of period 2 (Henson and Cushing 1997, Costantino et al. 1998, Henson et al. 1999). To analyze the period-4 habitat sequence used in the Jillson experiment we make a similar modification. To do so we assume, as in the previous studies, that each cannibalism coefficient in the deterministic LPA model is inversely proportional to flour volume (for an empirical test that supports this assumption see Costantino et al. 1998). If $c_{e a}$, $c_{e l}$, and $c_{p a}$ denote the coefficients in the $20 \mathrm{~g}$ habitat, then the deterministic "period-4 LPA model" for the periodically forced habitat becomes

$$
\begin{aligned}
L_{t+1}= & b A_{t} \exp \left(\frac{-c_{e a} A_{t}-c_{e l} L_{t}}{1+\alpha[\cos (\pi t / 2)+\sin (\pi t / 2)]}\right) \\
P_{t+1}= & \left(1-\mu_{l}\right) L_{t} \\
A_{t+1}= & P_{t} \exp \left(\frac{-c_{p a} A_{t}}{1+\alpha[\cos (\pi t / 2)+\sin (\pi t / 2)]}\right) \\
& +\left(1-\mu_{a}\right) A_{t}
\end{aligned}
$$

with $0 \leq \alpha<1$. Here $\alpha$ is the relative amplitude of the forcing oscillation. Thus, for example, in the experimental regime used by Jillson (1980) we have $\alpha=0.6$. The trigonometric functions are used to accommodate the habitat sequence. For $t=0,1,2,3$ and $\alpha=0.6$ we obtain from these functions the sequence 1.6, 1.6, 0.4, 0.4 for the denominators in Eqns. (2a) and (2c) which corresponds to a flour volume sequence of $32 \mathrm{~g}, 32 \mathrm{~g}, 8 \mathrm{~g}$, $8 \mathrm{~g}$ respectively. Note the autonomous model Eqns. (1a) $-(1 \mathrm{c})$ are obtained by setting $\alpha=0$ in the period -4 model Eqns. (2a)-(2c).

Validation of the period-4 LPA model is not a primary objective of this report. Nevertheless, we want to point out that not only are the data sets for parameter estimation distinct from the data sets to be analyzed, but the biological and mathematical context of the parameter estimation procedure (constant 20g habitat, autonomous LPA model) is distinct from the application (period-4 habitat, non-autonomous LPA model). Therefore, dynamical assertions based on the model (2a) $-(2 c)$ are pure predictions and are not fits to the Jillson data.

\section{Multiple attractor hypothesis}

Together with the parameter estimates given in "The model" section, the period-4 model (2a)-(2c) with $\alpha=$ 0.6 predicts two distinct, locally stable 4 -cycles and an 
Table 1. Model-predicted coordinates (rounded to four significant digits) of the two locally stable 4-cycles and the unstable 4-cycle.

\begin{tabular}{lcccc}
\hline Stable cycle A & 1st 32g habitat & 2nd 32g habitat & 1st 8g habitat & 2nd 8g habitat \\
\hline L-stage & $1.430 \times 10^{-2}$ & 232.4 & 0.2678 & 71.43 \\
P-stage & 42.23 & $8.457 \times 10^{-3}$ & 137.4 & 0.1583 \\
A-stage & 71.01 & 87.14 & 73.96 & 83.65 \\
Stable cycle B & 75.64 & 24.41 & 99.95 & $4.993 \times 10^{-4}$ \\
L-stage & $2.952 \times 10^{-4}$ & 44.73 & 14.43 & 59.10 \\
P-stage & 69.61 & 59.08 & 80.84 & 70.45 \\
A-stage & 30.92 & 73.69 & 19.47 & 8.189 \\
Unstable cycle & 4.837 & 18.28 & 43.58 & 11.51 \\
L-stage & 50.46 & 46.35 & 52.95 & 56.25 \\
P-stage & & &
\end{tabular}

unstable (saddle) 4-cycle (Table 1). Time series plots of the larval component of these three 4-cycles appear in Fig. 2. We note the following distinguishing features of these 4-cycles. Relative to the $32 \mathrm{~g}-32 \mathrm{~g}-8 \mathrm{~g}-8 \mathrm{~g}$ oscillation of the flour volume, the larval numbers in the stable 4-cycle denoted by A in Table 1 show a "low-high-lowhigh" pattern with the highest peak occurring in the second $32 \mathrm{~g}$ habitat. In relation to this pattern, the oscillatory pattern of larval numbers in cycle B is out-of-phase, showing a "high-low-high-low" pattern with the highest peak occurring in the first $8 \mathrm{~g}$ habitat. The larval numbers in the saddle 4-cycle, while peaking in the second $32 \mathrm{~g}$ habitat together with cycle $\mathrm{A}$, have a significantly different pattern from those of either stable 4-cycle. Instead of a repetitious "high-low" pattern, the saddle 4-cycle decreases from its peak for two consecutive time units after which it increases for two consecutive time units.

In addition to time series, we will use two additional conceptual tools from dynamical systems theory to visualize and tightly connect the model predictions with the data. The first concept is that of a composite map. Orbits of nonautonomous models such as the periodic LPA model should not be visualized in a three-dimensional, Euclidean coordinate system, or "phase space", since each triple $(L, P, A)$ (i.e. each point in the space) must also be identified with the time at which an orbit is at this point in order to specify the future of the orbit. This is because in model $(2 a)-(2 c)$, the state of the system at time $t+1$ depends explicitly on $t$ as well as on the values of $L_{t}, P_{t}$, and $A_{t}$. However, if the model is composed with itself three times, so that orbits correspond to every fourth step of the period-4 model, we obtain an autonomous composite map whose phase portrait facilitates visualization of both model predictions and the data (Henson et al. 1998, 1999). Actually, there are four different composite maps, each of whose orbits correspond to one of $t=0,4,8, \ldots$ or $t=1,5,9$, $\ldots$ or $t=2,6,10, \ldots$ or $t=3,7,11, \ldots$ in the period- 4 model. Each stable (unstable) 4-cycle of the period-4 model corresponds to a stable (unstable) fixed point of a composite model. Thus, each of the four composite models has two stable fixed points (one for each of the two stable 4-cycles) and one unstable fixed point (corresponding to the saddle 4-cycle), all of which can be visualized in the three-dimensional $(L, P, A)$ phase space.

Now that we can visualize the data and predictions in phase space, we turn to the second concept - basins of attraction. In a phase space, the set of all points which give rise to model orbits approaching an attractor is

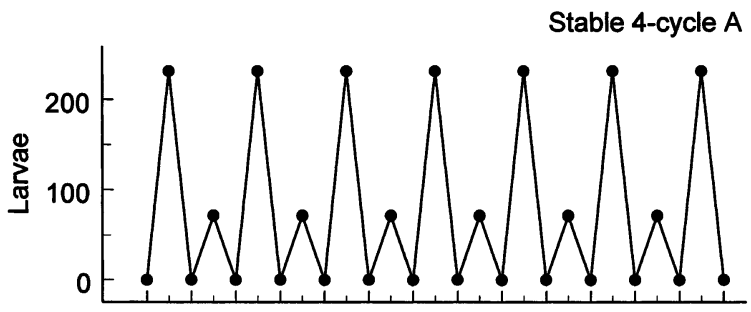

$\begin{array}{llllllllllllll}18 & 22 & 26 & 30 & 34 & 38 & 42 & 46 & 50 & 54 & 58 & 62 & 66 & 70\end{array}$

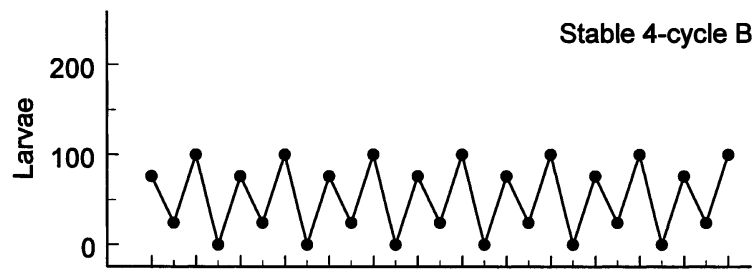

$\begin{array}{llllllllllllll}18 & 22 & 26 & 30 & 34 & 38 & 42 & 46 & 50 & 54 & 58 & 62 & 66 & 70\end{array}$

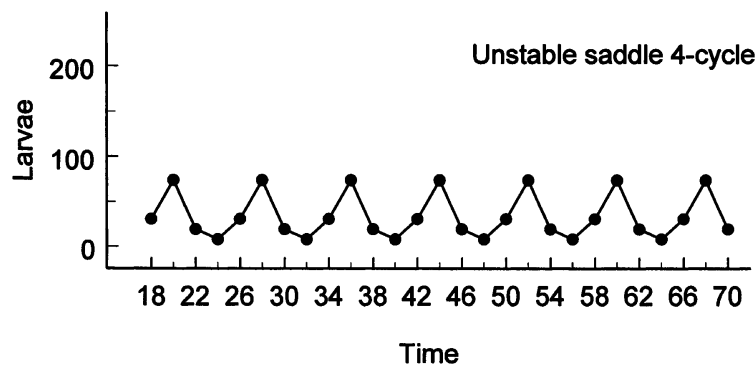

Fig. 2. The upper two plots show the larval time series for the two stable 4-cycles (A and B in Table 1) of the deterministic LPA model given by Eqns. (1a)-(1c) with the parameter values given in "The model" section. The bottom plot shows the larval time series of the unstable (saddle) 4-cycle. 


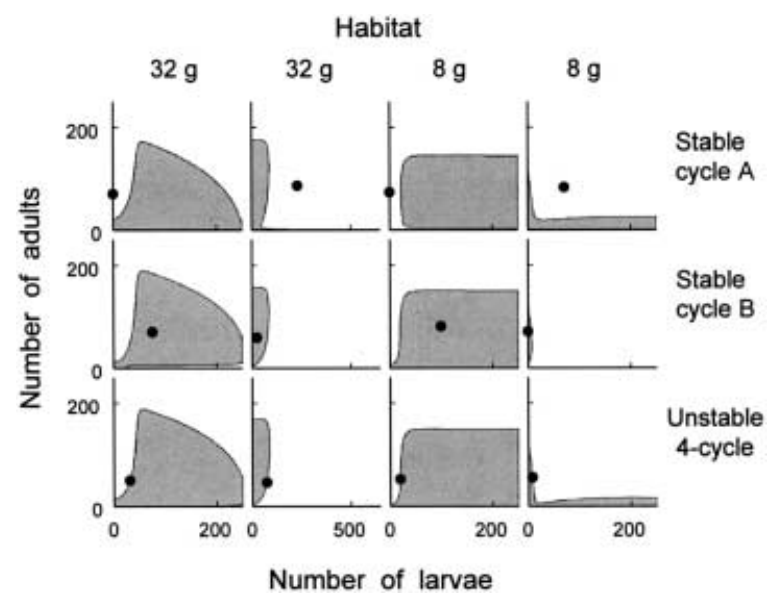

Fig. 3. Deterministic LPA period-4 model predictions with $\alpha=0.6$ and the parameter estimates given in "The model" section are displayed by means of cross-sections in phase space. In the first row of graphs the coordinates of the stable cycle A are plotted as solid circles in the two-dimensional cross-sections of phase space taken at the pupal coordinate plane. Similar plots appear in the second row of graphs for the 4-cycle B. In each graph the white area is the cross-section of the basin of attraction for 4-cycle A, and the shaded area is the cross-section of the basin of attraction for 4-cycle B. In the third row of graphs one sees, again in cross-sections taken at the pupal coordinate, the unstable 4-cycle points, which necessarily lie on the boundary that separates the attracting basins of the 4-cycles A and B.

called the basin of attraction of that attractor. Each of the two stable fixed points in a composite map has a three-dimensional basin of attraction in phase space. The saddle 4-cycle lies on the boundary separating these basins of attraction.

Three dimensional regions are difficult to draw and visualize. In order to facilitate our study in phase space, we present two-dimensional cross-sections in selected P-stage planes. For example, consider the composite map associated with the first $32 \mathrm{~g}$ habitat, that is, the one tracking times $t=0,4,8, \ldots$. In Fig. 3 the first column of graphs corresponds to this map. The first graph from this column shows the fixed point corresponding to stable cycle $\mathrm{A}$, and its basin of attraction, in a cross-section taken at the pupal coordinate $P=$ 42.23 of the fixed point (see Table 1). The second graph in the first column shows the fixed point corresponding to stable cycle B, and its basin of attraction, in a cross-section taken at the pupal coordinate $P=$ $2.952 \times 10^{-4}$ of that fixed point. The third graph in the first column shows the unstable fixed point, lying on the boundary separating the basins of attraction for cycles $\mathrm{A}$ and $\mathrm{B}$, in a cross-section taken at its pupal coordinate $P=4.837$. The graphs from the second column of graphs in Fig. 3 are interpreted similarly for the composite associated with the second $32 \mathrm{~g}$ habitat and corresponding to times $t=1,5,9, \ldots$. When read from left to right, the three rows of graphs in Fig. 3 show the temporal sequence corresponding to the stable cycle A, the stable cycle $\mathrm{B}$, and the unstable cycle respectively.

The format of graphs presented in Fig. 3 to visualize the model predicted cycles also provides a powerful way to visualize and study data. In Fig. 4 , each data point $\left(L^{*}, P^{*}, A^{*}\right)$ of culture A from the experiment is presented in the $P=P^{*}$ plane of the appropriate composite phase space, along with the cross-section of the basins of attraction. One can visualize the complete time series by reading the graphs from left to right across rows in descending order. On the other hand, one can see the time series for each of the four composite maps by reading down the columns. Fig. 5 presents culture B in the same format.

The phase space plots in Fig. 4 and 5 show that at week 18 culture A lay in the basin of attraction of cycle

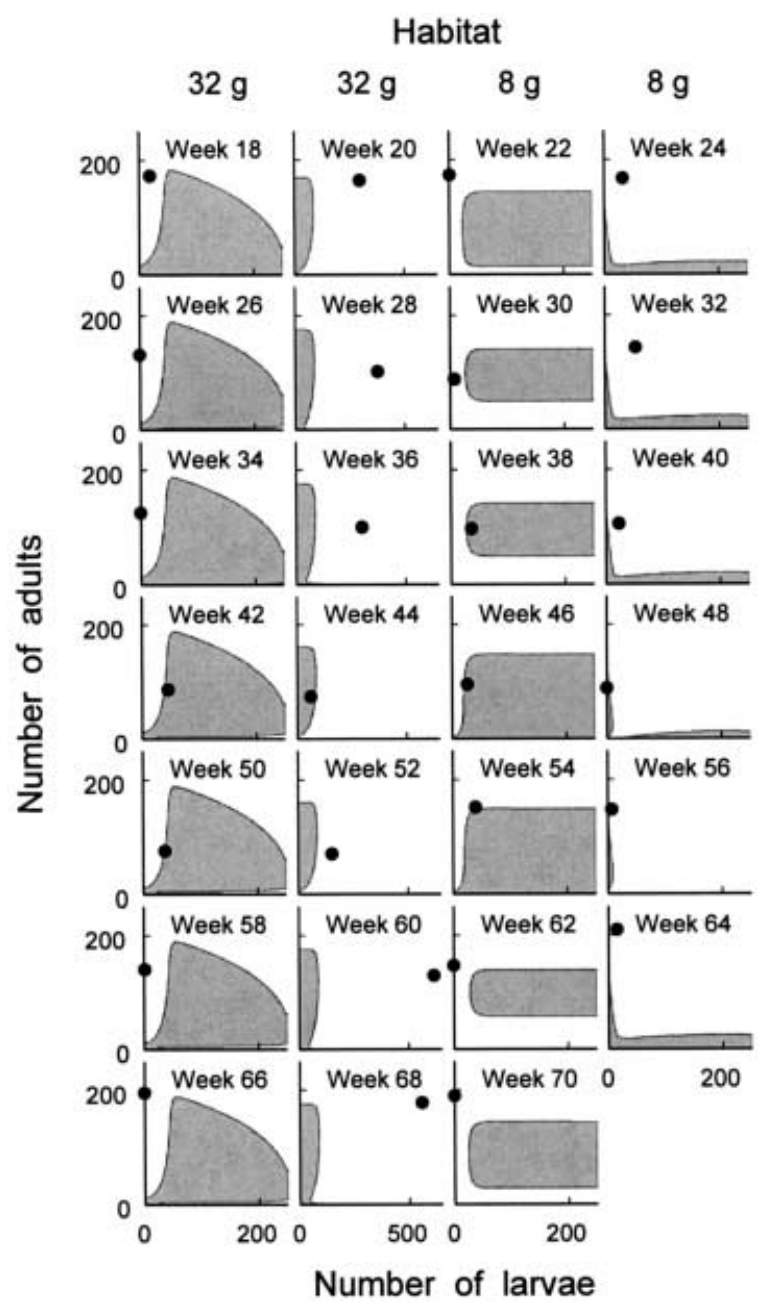

Fig. 4. Observed $(L, P, A)$ data for culture A are plotted in composite phase space using the same format as in Fig. 3. The movement of the data orbit to the basin boundary, from week 42 through week 56, corresponds to the influence of the unstable (saddle) 4-cycle noted during the same interval in the time series graph shown in Fig. 2. 


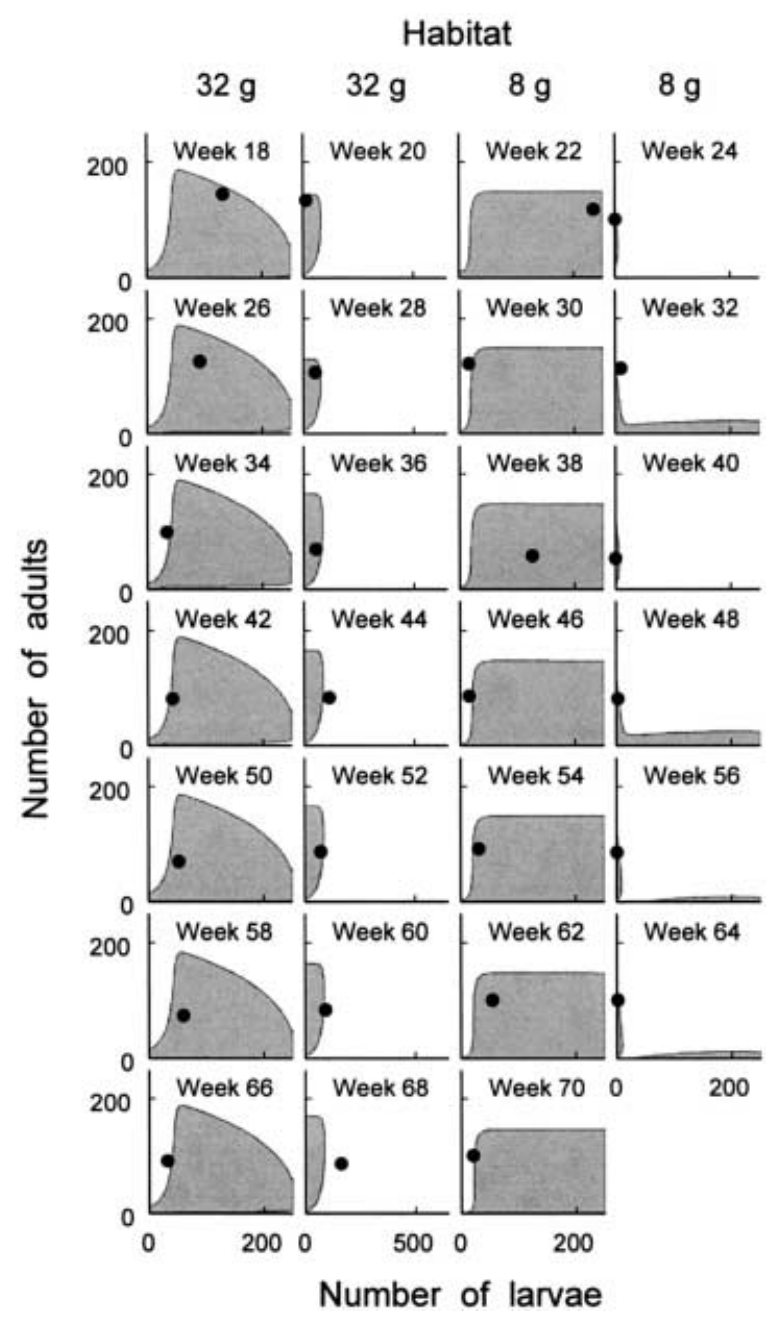

Fig. 5. Observed $(L, P, A)$ data for culture B are plotted in composite phase space using the same format as in Fig. 3. The $(L, P, A)$ data triples consistently remain in or close to the basin of attraction of stable cycle B, although late in the experiment (week 68) there appears to be a change to the basin of stable cycle A.

A, while culture B lay in the basin of attraction of cycle B. Furthermore, we see that culture A remained in the basin of attraction of cycle A until week 38 and culture $\mathrm{B}$ remained in the basin of attraction of cycle $\mathrm{B}$ until week 30. This explains the initial differences between the oscillatory patterns observed in the larval components of these two cultures, as described in "The data" section.

The multiple attractor hypothesis also explains the later temporal patterns seen in both cultures. As observed in "The data" section, the initial oscillatory patterns of both cultures are interrupted by interludes (from week 42 to 56 in culture A and week 28 to 66 in culture B) during which each culture displays an oscillatory pattern distinctly different from those of the two stable 4-cycles. In fact, during these interludes both cultures (Fig. 1) lie very close to the basin boundary (Fig. 4 and 5) and display the oscillatory pattern of the model predicted unstable 4-cycle (Fig. 2 and 3). Thus, within these time periods the saddle 4-cycle is exerting a greater influence than either of the stable 4-cycles. We refer to this as a "saddle fly-by". By the end of the experiment both cultures lie in the basin of attraction of 4-cycle A (Fig. 4 and 5) and, as a result, have become synchronized with the oscillatory pattern of this 4-cycle (Fig. 1 and 2). We say culture B "switched basins".

The concepts of basin switching and saddle fly-by's complete the explanation of the data. In a completely deterministic system, cultures cannot change from one basin of attraction to another; initial conditions completely set the long-term outcome. Attractor basin switching, such as occurred in culture B, happens because of stochasticity. In some cases, a stochastic event can place a culture directly into another attractor basin (Henson et al. 1998). In other cases, such as reported here, the basin switching is associated with a saddle fly-by (Henson et al. 1999).

A saddle fly-by occurs when a stochastic occurrence places a data point near the saddle (or near its stable manifold). As the culture "flies by" the saddle and returns to an attractor, the transient dynamics exhibit characteristics of the saddle. One characteristic of a saddle is that nearby orbits can separate and approach different attractors. Thus, when an orbit is near a saddle (or more generally near the boundary separating the basins of attraction of the stable cycles A and B) randomness plays a large role in determining the outcome of the fly-by. (This is reminiscent of the saddle in the Lotka-Volterra model for competing species and the seminal work of Leslie et al. 1968.)

Both saddle fly-by's and basin switching involve properties of the deterministic LPA model as well as stochasticity; therefore, it is important to examine the impact of noise on the dynamic behavior of the deterministic LPA model. Repeated simulations of the demographic stochastic LPA model starting from the same initial conditions produce different time series predictions. Simulations starting from either the location of culture A at week 18 (namely, $(L, P, A)=(19,14,173)$ ) or culture B at week $18((L, P, A)=133,5,145))$ typically show a mix of patterns characteristic of the three 4-cycles, involving one or more saddle cycle fly-by's. The outcome of a saddle fly-by is sometimes a switch in attractor basins, but not always. Two simulations of the demographic stochastic LPA model (with parameter estimates given in "The model" section) appear in Fig. 6. These two examples bear resemblance to the larval time series of culture A and culture B (Fig. 1).

\section{Discussion}

In this paper we have studied data from an experiment of Jillson (1980) in which cultures of Tribolium were 
grown in a periodically oscillating flour volume with period 4 ( 8 weeks). We used the concepts of composite maps, basins of attraction, basin switching, and saddle fly-by's to make an ecological hypothesis concerning the existence of multiple attractors in a periodically forced system more accessible to experimental scrutiny. The period-4 LPA model's prediction of two stable 4-cycles and one unstable (saddle) 4-cycle for Jillson's experiment requires the data to match very specific geometric phase space details of the basins of attraction forecast by the model. If the observations are to be considered consistent with the multiple attractor hypothesis, the data must fall in the appropriate basins in the appropriate sequence. In addition, the time series of the data must show the oscillatory characteristics associated with the attractor of each basin. Although these are very demanding criteria, we have seen that the data support the multiple attractor hypothesis.

Basin switching and the influence of saddles has been documented, by means of the LPA model, in several different Tribolium experiments (Cushing et al. 1998a, b, Henson et al. 1998, 1999). For an example of basin switching in physics, see Poon and Grebogi (1995).

A consequence of noise is an increase in the repertoire of time series orbits: each of the locally stable attractors can be visited along with the unstable invariant sets which under strict deterministic theory may have little or no impact on population time series. The signatures of all these deterministic entities potentially become part of the observed stochastic time series records (Earn et al. 2000). Without the benefit of an understanding of the underlying deterministic and stochastic forces, such time series are very difficult to interpret (Kaitala et al. 1999, 2000).

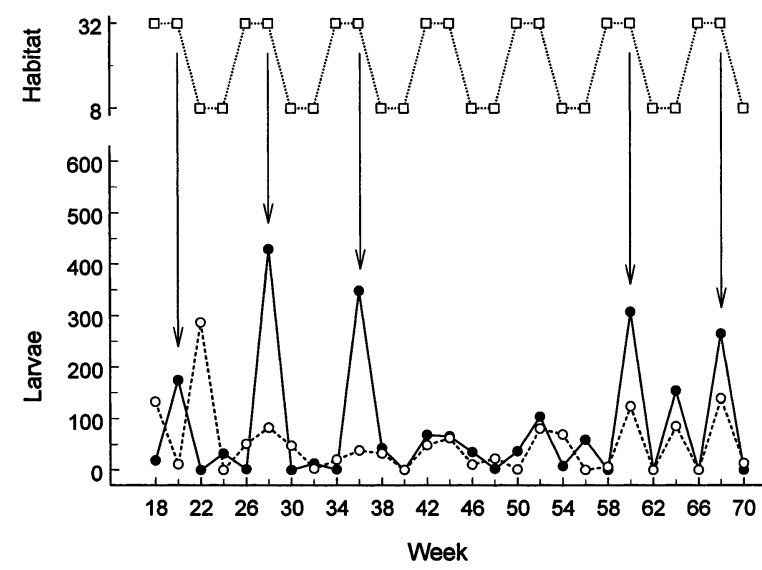

Fig. 6. The solid circles show a simulation of the demographic stochastic LPA model that begins (at week 18) at the same initial condition as culture A shown in Fig. 1. The open circles show a simulation that begins (at week 18) at the same initial condition as culture B in Fig. 1. Note the similarity between these simulations and the data plots in Fig. 1.
Discrete models of populations with stable inherent oscillations in constant habitats predict multiple attracting oscillatory final states in the presence of habitat periodicity (Henson 2000). Suppose an autonomous map with an inherent stable $q$-cycle is periodically forced with period $r$. In the absence of forcing, the inherent $q$-cycle attractor consists of $q$ out-of-phase stable $q$-cycle solutions. Henson (2000) shows that in the advent of periodic forcing, these solutions (generically) are perturbed into $q$ out-of-phase stable cycles with minimal period equal to the least common multiple $(\mathrm{lcm})$ of $q$ and $r$. The number of cycles which are distinct modulo phase differences is equal to the greatest common divisor ( $\mathrm{gcd})$ of $q$ and $r$; that is, $q \operatorname{lcm}(q, r)$-cycles exist on $\operatorname{gcd}(q, r)$ attractors. The ecological translation of the above mathematical statement leads to the identification of many testable hypotheses. For example, in the beetle system with an inherent stable $q=2$-cycle with a habitat forced with period $r=3$ we expect two out-of-phase $\operatorname{lcm}(2,3)=6$-cycles on $\operatorname{gcd}(2,3)=1$ attractor (that is, the two 6-cycles will be phase shifts of each other). The theorem is relevant to this paper: in a population with an inherent stable $q=2$-cycle with a habitat forced with period $r=4$ we expect two out-ofphase $\operatorname{lcm}(2,4)=4$-cycles on $\operatorname{gcd}(2,4)=2$ different attractors.

Given the generality of this mathematical prediction we anticipate that other laboratory systems will show similar results in the presence of periodic environmental forcing. For example, recent studies have found stable inherent oscillations in laboratory populations of phycitine moths (Plodia interpunctella) (Briggs et al. 2000). Populations of these animals placed in an environment forced with a certain period should, we predict, yield complex cycle patterns with multiple attractors.

In closing, we return to a question posed by May (1977): Are the dynamics of local natural populations described by a global attractor or, rather, composed of many locally stable attractors? Our analysis here is based on a mathematical model and a laboratory experiment; consequently, we address this question with caution. Nevertheless, the ease with which the mathematical model leads to multiple attractors is informative. The laboratory confirmation of the mathematical model reinforces the suggestion that natural populations have the potential to display the properties of multiple attractors. With the latter in mind, time series observations from natural populations may well contain the features of several different attractors, as well as unstable invariant sets, that make up a multiple attractor system.

Acknowledgements - Thanks to David Jillson for making the data from his 1980 experiment available to us. This work was supported in part by grants from the US National Science Foundation (DMS 9973126, DMS 9981374, DMS 9981423 DMS 9981458) and a Howard Hughes Medical Institute grant to the College of William and Mary (S. M. Henson). Sheree LaVarge participated in the project as an undergraduate research student. 


\section{References}

Briggs, C. J., Sait, S. M., Begon, M. et al. 2000. What causes generation cycles in populations of stored-product moths? J. Anim. Ecol. 69: 352-366.

Caswell, H. 2000. Matrix population models: construction analysis, and interpretation, 2nd edition. - Sinauer Associates, Inc.

Costantino, R. F., Cushing, J. M., Dennis, B. et al. 1998. Resonant population cycles in temporally fluctuating habitats. - Bull. Math. Biol. 60: 247-273.

Cushing, J. M., Dennis, B., Desharnais, R. A. and Costantino, R. F. 1998a. Moving toward an unstable equilibrium: saddle nodes in population systems. - J. Anim. Ecol. 67 298-306.

Cushing, J. M., Costantino, R. F., Dennis, B. et al. 1998b. Nonlinear population dynamics: models, experiments, and data. - J. Theor. Biol. 194: 1-9.

Desharnais, R. A. and Costantino, R. F. 1980. Genetic analysis of a population of Tribolium. VII. Stability: response to genetic and demographic perturbations. - Can. J. Gen. Cytol. 22: 577-589.

Dennis, B., Desharnais, R. A., Cushing, J. M. and Costantino, R. F. 1995. Non-linear demographic dynamics: mathematical models, statistical methods and biological experiments. - Ecol. Monogr. 65: 261-281.

Dennis, B., Desharnais, R. A., Cushing, J. M. and Costantino, R. F. 1997. Transitions in population dynamics: equilibria to periodic cycles to aperiodic cycles. - J. Anim. Ecol. 66 $704-729$.

Dennis, B., Desharnais, R. A., Cushing, J. M. et al. 2001. Estimating chaos and complex dynamics in an insect population. - Ecol. Monogr. 71: 277-303.

Earn, D. J. D., Rohani, P., Bolker, B. M. and Grenfell, B. T. 2000. A simple model for complex dynamical transitions in epidemics. - Science 287: 667-670.

Henson, S. M. 2000. Multiple attractors and resonance in periodically-forced population models. - Physica D: Nonlinear Phenomena 140: 33-49.
Henson, S. M. and Cushing, J. M. 1997. The effect of periodic habitat fluctuations on a nonlinear insect population model. - J. Math. Biol. 36: 201-226.

Henson, S. M., Cushing, J. M., Costantino, R. F. et al. 1998. Phase switching in population cycles. - Proc. R. Soc. Lond. B 265: 2229-2234.

Henson, S. M., Costantino, R. F., Cushing, J. M. et al. 1999. Multiple attractors, saddles, and population dynamics in periodic habitats. - Bull. Math. Biol. 61: 1121-1149.

Jillson, D. A. 1980. Insect populations respond to fluctuating environments. - Nature 288: 699-700.

Kaitala, V., Ylikarjula, J. and Heino, M. 1999. Dynamic complexities in host-parasitoid interaction. - J. Theor. Biol. 197: $331-341$.

Kaitala, V., Ylikarjula, J. and Heino, M. 2000. Non-unique population dynamics: basic patterns. - Ecol. Modelling 135: $127-134$.

King, A. A. and Schaffer, W. M. 1999. The rainbow bridge: Hamiltonian limits and resonance in predator-prey dynamics. - J. Math. Biol. 39: 439-469.

Kot, M. and Schaffer, W. M. 1984. The effects of seasonality on discrete models of population growth. - Theor. Pop. Biol. 26: 340-360.

Leslie, P. H., Park, T. and Mertz, D. B. 1968. The effect of varying the initial numbers on the outcome of competition between two Tribolium species. - J. Anim. Ecol. 37: 9-28.

May, R. M. 1977. Thresholds and breakpoints in ecosystems with a multiplicity of stable states. - Nature 269: 471-477.

Mueller, L. D. and Joshi, A. 2000. Stability in model populations. - Princeton University Press.

Poon, L. and Grebogi, C. 1995. Controlling complexity. - Phys. Rev. Lett. 75: 4023-4026.

Roberds, J. H. and Selgrade, J. F. 2000. Dynamical analysis of density-dependent selection in a discrete migration model. Math. Biosci. 164: 1-15.

Selgrade, J. F. and Roberds, J. H. 2001. On the structure of attractors for discrete, periodically forced systems with applications to population models. - Physica D 158: 69-82.

Vandermeer, J. and Yodzis, P. 1999. Basin boundary collision as a model of discontinuous change in ecosystems. - Ecology 80: 1817-1827. 